\title{
Synthesis, Characterization and Antimicrobial Activity of Some Thiazole Derivatives
}

\author{
I. SINGH \\ Department of Chemistry, Janta Vedic PG College Baraut (Baghpat) U.P-250611, India \\ drarunmrt@gmail.com
}

Received 6 November 2014 / Accepted 28 November 2014

\begin{abstract}
A series of $N$-(4-(3-acetamidophenyl)thiazol-2-yl)-2 (substituted phenylamino)acetamide (4-10) were prepared by the reaction of $\mathrm{N}$-(4-(3-acetamidophenyl)thiazol-2-yl)-2-chloroacetamide with substituted aniline. All the newly synthesized compounds were screened for their antimicrobial activity and compared with standard drug ciprofloxacin and fluconazole against different bacteria and fungi respectively. The structure of all the compounds were established by the elemental $(\mathrm{C}, \mathrm{H}, \mathrm{N})$ and spectral (IR and ${ }^{1} \mathrm{H}$ NMR) analysis.
\end{abstract}

Keywords: Thiazole, Antibacterial activity, Antifungal activity, Ciprofloxacin, Fluconazole

\section{Introduction}

The synthesis of condensed thiazole heterocycles has been explored to a maximum extent owing to their association with wide spectrum of pharmacological activities such as antimicrobial $^{1-5}$, antibacterial ${ }^{6,7}$, antifungal ${ }^{8}$, anticonvulsant $^{9,10}$, anti-inflammatory ${ }^{11}$, antitumor $^{12}$ and anticancer ${ }^{13}$ etc. Encouraged by these facts and in continuation of our research program, synthesis of pharmacologically interesting thiazole derivatives it was thought worthwhile to synthesized some new substituted thiazole derivatives with the hope to get better antimicrobial agents.

\section{Experimental}

The melting points of compounds were determined in open capillaries with the help of thermonic melting point apparatus and were uncorrected. The homogeneity of newly synthesized compounds was routinely checked by thin layer chromatography (TLC). Elemental analysis $(\mathrm{C}, \mathrm{H}, \mathrm{N})$ of the synthesized compounds were determined by perkinElmer 2400 elemental analyzer, and results were found within the $\pm 0.4 \%$ of theoretical values (Table 1). The IR spectra were recorded on a Beckman Acculab-10 Spectrometer ( $v$ max in $\mathrm{cm}^{-1}$ ) and the ${ }^{1} \mathrm{H}$ NMR spectra were recorded by Brucker DPX-300MHz using $\mathrm{CDCl}_{3}$ as solvent (Table 2). The synthesis of the target compounds was accomplished according to the reaction sequence illustrated in Scheme 1. 


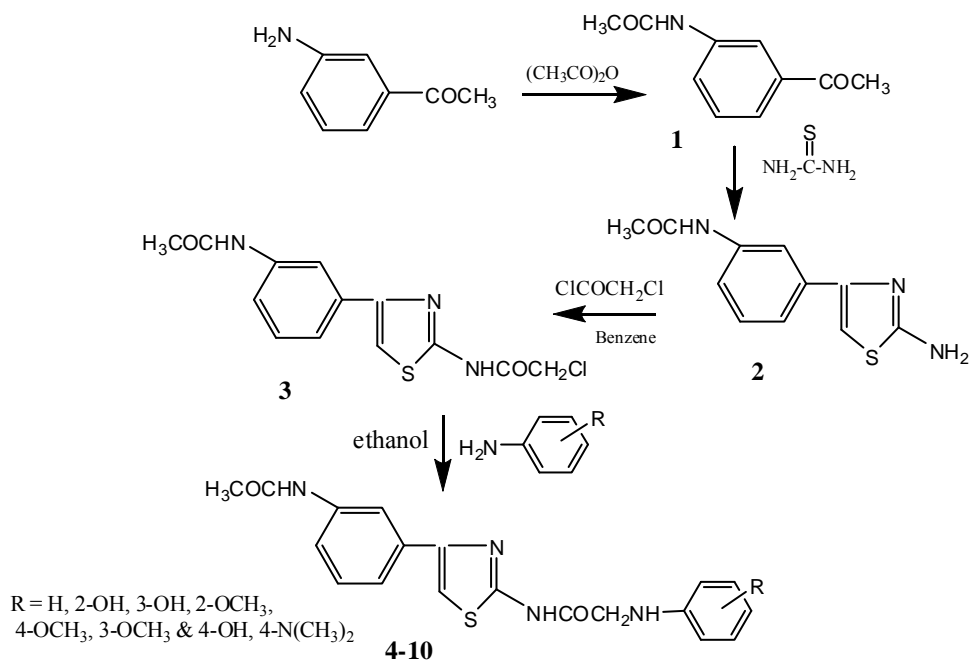

Scheme 1

\section{Synthesis of N-(3-acetylphenyl)acetamide (1)}

A mixture of 1-(3-aminophenyl)ethanone $(0.01 \mathrm{~mol})$ and acetic anhydride $(30 \mathrm{~mL})$ was refluxed for $2 \mathrm{~h}$ and the reaction mixture was cooled. The solid thus obtained was filtered, dried and recrystallized from ethanol to yield compound $\mathbf{1}$.

\section{Synthesis of N-(3-(2-aminothiazol-4-yl)phenyl)acetamide (2)}

A mixture of iodine $(0.03 \mathrm{~mol})$ and thiourea $(0.06 \mathrm{~mol})$ was triturated and the mixture poured into a conical flask containing $N$-(3-acetylphenyl)acetamide (1) $(0.03 \mathrm{~mol})$. The reaction mixture was heated for $8 \mathrm{~h}$. The solid obtained was washed with diethyl ether, after then it was washed with sodium thiosulphate. Finally, the reaction mixture was poured in ice water. The solid thus obtained was filtered, washed with water, dried and recrystallized from appropriate solvent to yield compound 2.

\section{Synthesis of N-(4-(3-acetamidophenyl)thiazol-2-yl)-2-chloroacetamide (3)}

The suspension of compound $2(0.01 \mathrm{~mol})$ in glacial acetic acid $(30 \mathrm{~mL})$, chloroacetyl chloride was added drop wise with constant stirring. The reaction mixture was refluxed gently at $120{ }^{\circ} \mathrm{C}$ for $6 \mathrm{~h}$ and poured on to the crushed ice. The solid mass so obtained was filtered, washed with ice cold water, dried and recrystallized from appropriate solvent to yield compound 3.

\section{Synthesis of N-(4-(3-acetamidophenyl)thiazol-2-yl)-2-(substitutedphenylamino) acetamide (4-10)}

To a mixture of compound $3(0.01 \mathrm{~mol})$ in methanol $(20 \mathrm{~mL})$ and in this solution substituted aniline were added in drop wise manner and mixture was refluxed for 4-6 h. After completion of the reaction it is checked by TLC. The excess of methanol was distilled off. The obtained solid residue was washed with petroleum ether $\left(40-60{ }^{0} \mathrm{C}\right)$ and recrystallized from acetone to give compounds 4-10.

\section{Antimicrobial activity}

All the newly synthesized compounds 2-10 were tested for their antimicrobial activity. The effects of unknown compounds were compared with the standard drug ciprofloxacin for bacteria 
and fluconazole for fungi. Antibacterial activity was performed against staphylococcus aureus, Escherischia coli, Proteus vulgaris and antifungal activity against Aspergillus niger, Aspergillus flavus and Candida krusei. The antibacterial activity was assayed by cup plate method $^{14}$ and antifungal activity was assayed by standard agar disc diffusion method ${ }^{15}$.

\section{Results and Discussion}

The newly synthesized compounds were tested for their antimicrobial activity against different microorganism. The results were discussed in Table 3. Compounds 8, 9 and $\mathbf{1 0}$ exhibiting good antibacterial activity against E. coli, S. aureus and $P$. vulgaris. The remaining compounds exhibited moderate activity against all the bacteria used for screening. In antifungal activity compounds $\mathbf{9}$ and $\mathbf{1 0}$ exhibited excellent activity against A. niger and $C$. krusai. Compound $\mathbf{7}$ showed good antifungal activity against different fungi and all remaining compounds exhibiting moderate activity against all the three organism used for screening.

Table 1. Physical and analytical data of the compounds 1-10

\begin{tabular}{|c|c|c|c|c|c|c|c|c|}
\hline \multirow[t]{2}{*}{ Compd. } & \multirow[t]{2}{*}{$\mathrm{R}$} & \multirow[t]{2}{*}{$\begin{array}{c}\text { Recrystalization } \\
\text { solvent }\end{array}$} & \multirow[t]{2}{*}{$\begin{array}{c}\text { Yield } \\
\% \\
\end{array}$} & \multirow[t]{2}{*}{$\begin{array}{c}\text { M.P } \\
{ }^{0} \mathrm{C} \\
\end{array}$} & \multirow[t]{2}{*}{ Mol. Formula } & \multicolumn{3}{|c|}{$\begin{array}{l}\text { Analysis \% found } \\
\text { (Calculated) }\end{array}$} \\
\hline & & & & & & $\mathrm{C}$ & $\mathrm{H}$ & $\mathrm{N}$ \\
\hline 1 & - & ethanol & 87 & 193 & $\mathrm{C}_{10} \mathrm{H}_{11} \mathrm{NO}_{2}$ & $\begin{array}{c}67.75 \\
(67.78\end{array}$ & $\begin{array}{l}6.25 \\
6.26\end{array}$ & $\begin{array}{c}7.92 \\
7.90)\end{array}$ \\
\hline 2 & - & Acetone & 84 & 211 & $\mathrm{C}_{11} \mathrm{H}_{11} \mathrm{~N}_{3} \mathrm{OS}$ & $\begin{array}{r}56.64 \\
(56.63\end{array}$ & $\begin{array}{l}4.72 \\
4.75\end{array}$ & $\begin{array}{c}18.03 \\
18.01)\end{array}$ \\
\hline 3 & - & Methanol & 80 & 189 & $\mathrm{C}_{13} \mathrm{H}_{12} \mathrm{ClN}_{3} \mathrm{O}_{2} \mathrm{~S}$ & $\begin{array}{c}50.42 \\
(50.40\end{array}$ & $\begin{array}{l}3.91 \\
3.90\end{array}$ & $\begin{array}{c}13.58 \\
13.56)\end{array}$ \\
\hline 4 & $\mathrm{H}$ & Ethanol & 82 & 226 & $\mathrm{C}_{19} \mathrm{H}_{18} \mathrm{~N}_{4} \mathrm{O}_{2} \mathrm{~S}$ & $\begin{array}{r}62.25 \\
(62.28\end{array}$ & $\begin{array}{l}4.96 \\
4.95\end{array}$ & $\begin{array}{l}15.26 \\
15.29)\end{array}$ \\
\hline 5 & $2-\mathrm{OH}$ & Methanol & 79 & 238 & $\mathrm{C}_{19} \mathrm{H}_{18} \mathrm{~N}_{4} \mathrm{O}_{3} \mathrm{~S}$ & $\begin{array}{r}59.69 \\
(59.67\end{array}$ & $\begin{array}{l}4.75 \\
4.74\end{array}$ & $\begin{array}{l}14.62 \\
14.65)\end{array}$ \\
\hline 6 & $3-\mathrm{OH}$ & Acetone & 74 & 235 & $\mathrm{C}_{19} \mathrm{H}_{18} \mathrm{~N}_{4} \mathrm{O}_{3} \mathrm{~S}$ & $\begin{array}{r}59.64 \\
(59.67\end{array}$ & $\begin{array}{l}4.73 \\
4.74\end{array}$ & $\begin{array}{l}14.67 \\
14.65)\end{array}$ \\
\hline 7 & $2-\mathrm{OCH}_{3}$ & Methanol & 71 & 247 & $\mathrm{C}_{20} \mathrm{H}_{20} \mathrm{~N}_{4} \mathrm{O}_{3} \mathrm{~S}$ & $\begin{array}{r}60.56 \\
(60.59\end{array}$ & $\begin{array}{l}4.04 \\
5.08\end{array}$ & $\begin{array}{c}14.14 \\
14.13)\end{array}$ \\
\hline 8 & $4-\mathrm{OCH}_{3}$ & Ethanol & 69 & 249 & $\mathrm{C}_{20} \mathrm{H}_{20} \mathrm{~N}_{4} \mathrm{O}_{3} \mathrm{~S}$ & $\begin{array}{r}60.58 \\
(60.59\end{array}$ & $\begin{array}{l}5.06 \\
5.08\end{array}$ & $\begin{array}{l}14.15 \\
14.13)\end{array}$ \\
\hline 9 & $\begin{array}{l}3-\mathrm{OCH}_{3} \\
\& 4-\mathrm{OH}\end{array}$ & Acetone & 64 & 265 & $\mathrm{C}_{20} \mathrm{H}_{20} \mathrm{~N}_{4} \mathrm{O}_{4} \mathrm{~S}$ & $\begin{array}{c}58.27 \\
(58.24\end{array}$ & $\begin{array}{l}4.90 \\
4.89\end{array}$ & $\begin{array}{c}13.54 \\
13.58)\end{array}$ \\
\hline 10 & $\mathrm{~N}\left(\mathrm{CH}_{3}\right)_{2}$ & Methanol & 57 & 253 & $\mathrm{C}_{21} \mathrm{H}_{23} \mathrm{~N}_{5} \mathrm{O}_{2} \mathrm{~S}$ & $\begin{array}{r}61.56 \\
(61.59\end{array}$ & $\begin{array}{l}5.69 \\
5.66\end{array}$ & $\begin{array}{c}17.13 \\
17.10)\end{array}$ \\
\hline
\end{tabular}

Table 2. Spectral data of compounds 1-10

\begin{tabular}{cll}
\hline Compd. & \multicolumn{1}{c}{ IR $(\mathrm{KBr}) v_{\max }$ in $\mathrm{cm}^{-1}$} & \multicolumn{1}{c}{${ }^{1} \mathrm{H}-\mathrm{NMR}\left(\mathrm{CDCl}_{3}+\mathrm{DMSOd}_{6}\right) \delta$ in ppm } \\
\hline 1 & $3355(\mathrm{NH}), 3038(\mathrm{C}-\mathrm{H}$ aromatic $)$, & $8.84(\mathrm{~s}, 1 \mathrm{H}, \mathrm{NH}), 7.13-8.12(\mathrm{~m}, 4 \mathrm{H}, \mathrm{Ar}-$ \\
& $1692(\mathrm{C}=\mathrm{O}), 1530(\mathrm{C}-\mathrm{C}$ of aromatic & $\mathrm{H}), 2.57\left(\mathrm{~s}, 3 \mathrm{H}, \mathrm{NHCOCH}_{3}\right), 2.25(\mathrm{~s}, 3 \mathrm{H}$, \\
& ring $), 1547(\mathrm{C}-\mathrm{N})$ & $\left.\mathrm{COCH}_{3}\right)$ \\
2 & $3362\left(\mathrm{NH}_{2}\right), 3031(\mathrm{C}-\mathrm{H}$ aromatic $)$, & $8.89\left(\mathrm{~s}, 2 \mathrm{H}, \mathrm{NH}_{2}\right), 8.47(\mathrm{~s}, 1 \mathrm{H}, \mathrm{NH}), 7.70$ \\
& $1695(\mathrm{C}=\mathrm{O}), 1680(\mathrm{C}=\mathrm{N}), 1538(\mathrm{C}-\mathrm{C}$ & $(\mathrm{s}, 1 \mathrm{H}, \mathrm{CH}$ thiazole $), 7.12-8.15(\mathrm{~m}, 4 \mathrm{H}$, \\
& of aromatic ring $), 1061(\mathrm{C}-\mathrm{S}-\mathrm{C})$ & Ar-H $), 2.59\left(\mathrm{~s}, 3 \mathrm{H}, \mathrm{NHCOCH}_{3}\right)$ \\
\hline
\end{tabular}




\begin{tabular}{|c|c|c|}
\hline 3 & $\begin{array}{l}3354(\mathrm{NH}), 3037(\mathrm{C}-\mathrm{H} \text { aromatic }), \\
1689(\mathrm{C}=\mathrm{N}), 1536(\mathrm{C}-\mathrm{C} \text { of aromatic } \\
\text { ring }), 1065(\mathrm{C}-\mathrm{S}-\mathrm{C})\end{array}$ & $\begin{array}{l}8.85(\mathrm{~s}, 2 \mathrm{H}, 2 \mathrm{X} \mathrm{NH}), 7.53(\mathrm{~s}, 1 \mathrm{H}, \mathrm{CH}), \\
7.15-8.14(\mathrm{~m}, 4 \mathrm{H}, \mathrm{Ar}-\mathrm{H}), 3.53(\mathrm{~s}, 2 \mathrm{H}, \\
\left.\mathrm{CH}_{2}\right), 2.54\left(\mathrm{~s}, 3 \mathrm{H}, \mathrm{CH}_{3}\right)\end{array}$ \\
\hline 4 & $\begin{array}{l}3358(\mathrm{NH}), 3039(\mathrm{C}-\mathrm{H} \text { aromatic }), \\
1686(\mathrm{C}=\mathrm{N}), 1531(\mathrm{C}-\mathrm{C} \text { of aromatic } \\
\text { ring }), 1060(\mathrm{C}-\mathrm{S}-\mathrm{C})\end{array}$ & $\begin{array}{l}8.85(\mathrm{~s}, 3 \mathrm{H}, 3 \mathrm{X} \mathrm{NH}), 8.48(\mathrm{~s}, 1 \mathrm{H}, \mathrm{CH}), \\
7.13-8.15(\mathrm{~m}, 9 \mathrm{H}, \mathrm{CH}-\mathrm{Ar}), 3.55(\mathrm{~s}, 2 \mathrm{H}, \\
\left.\mathrm{CH}_{2}\right), 2.56\left(\mathrm{~s}, 3 \mathrm{H}, \mathrm{CH}_{3}\right)\end{array}$ \\
\hline 5 & $\begin{array}{l}3476(\mathrm{OH}), 3356(\mathrm{NH}), 3036(\mathrm{C}-\mathrm{H} \\
\text { aromatic }), 1684(\mathrm{C}=\mathrm{N}), 1535(\mathrm{C}-\mathrm{C} \\
\text { of aromatic ring }), 1065(\mathrm{C}-\mathrm{S}-\mathrm{C})\end{array}$ & $\begin{array}{l}12.49(\mathrm{~s}, 1 \mathrm{H}, \mathrm{OH}), 8.83(\mathrm{~s}, 3 \mathrm{H}, 3 \mathrm{X} \mathrm{NH}) \\
8.43(\mathrm{~s}, 1 \mathrm{H}, \mathrm{CH}), 7.14-8.13(\mathrm{~m}, 8 \mathrm{H}, \mathrm{CH}- \\
\mathrm{Ar}), 3.52\left(\mathrm{~s}, 2 \mathrm{H}, \mathrm{CH}_{2}\right), 2.55\left(\mathrm{~s}, 3 \mathrm{H}, \mathrm{CH}_{3}\right)\end{array}$ \\
\hline 6 & $\begin{array}{l}3473(\mathrm{OH}), 3358(\mathrm{NH}), 3037(\mathrm{C}-\mathrm{H} \\
\text { aromatic }), 1688(\mathrm{C}=\mathrm{N}), 1531(\mathrm{C}-\mathrm{C} \\
\text { of aromatic ring }), 1061(\mathrm{C}-\mathrm{S}-\mathrm{C})\end{array}$ & $\begin{array}{l}12.45(\mathrm{~s}, 1 \mathrm{H}, \mathrm{OH}), 8.81(\mathrm{~s}, 3 \mathrm{H}, 3 \mathrm{X} \mathrm{NH}) \\
8.47(\mathrm{~s}, 1 \mathrm{H}, \mathrm{CH}), 7.12-8.14(\mathrm{~m}, 8 \mathrm{H}, \mathrm{CH}- \\
\mathrm{Ar}), 3.56\left(\mathrm{~s}, 2 \mathrm{H}, \mathrm{CH}_{2}\right), 2.52\left(\mathrm{~s}, 3 \mathrm{H}, \mathrm{CH}_{3}\right)\end{array}$ \\
\hline 7 & $\begin{array}{l}3355(\mathrm{NH}), 3042(\mathrm{C}-\mathrm{H} \text { aromatic }), \\
1688(\mathrm{C}=\mathrm{N}), 1670(\mathrm{C}=\mathrm{O}), 1532(\mathrm{C}-\mathrm{C} \\
\text { of aromatic ring }), 1063(\mathrm{C}-\mathrm{S}-\mathrm{C})\end{array}$ & $\begin{array}{l}8.89(\mathrm{~s}, 3 \mathrm{H}, 3 \mathrm{X} \mathrm{NH}), 8.49(\mathrm{~s}, 1 \mathrm{H}, \mathrm{CH}) \\
7.11-8.13(\mathrm{~m}, 8 \mathrm{H}, \mathrm{CH}-\mathrm{Ar}),\left(\mathrm{s}, 3 \mathrm{H}, \mathrm{OCH}_{3}\right) \\
3.55\left(\mathrm{~s}, 2 \mathrm{H}, \mathrm{CH}_{2}\right), 2.50\left(\mathrm{~s}, 3 \mathrm{H}, \mathrm{CH}_{3}\right)\end{array}$ \\
\hline 8 & $\begin{array}{l}3350(\mathrm{NH}), 3039(\mathrm{C}-\mathrm{H} \text { aromatic }), \\
1689(\mathrm{C}=\mathrm{N}), 1675(\mathrm{C}=\mathrm{O}), 1536(\mathrm{C}-\mathrm{C} \\
\text { of aromatic ring }), 1061(\mathrm{C}-\mathrm{S}-\mathrm{C})\end{array}$ & $\begin{array}{l}8.84(\mathrm{~s}, 3 \mathrm{H}, 3 \mathrm{X} \mathrm{NH}), 8.44(\mathrm{~s}, 1 \mathrm{H}, \mathrm{CH}) \\
7.10-8.15(\mathrm{~m}, 8 \mathrm{H}, \mathrm{CH}-\mathrm{Ar}), 4.06(\mathrm{~s}, 3 \mathrm{H}, \\
\left.\mathrm{OCH}_{3}\right), 3.43\left(\mathrm{~s}, 2 \mathrm{H}, \mathrm{CH}_{2}\right), 2.56\left(\mathrm{~s}, 3 \mathrm{H}, \mathrm{CH}_{3}\right)\end{array}$ \\
\hline 9 & $\begin{array}{l}3479(\mathrm{OH}), 3353(\mathrm{NH}), 3036(\mathrm{C}-\mathrm{H} \\
\text { aromatic }), 1683(\mathrm{C}=\mathrm{N}), 1678(\mathrm{C}=\mathrm{O}), \\
1535(\mathrm{C}-\mathrm{C} \text { of aromatic ring }), 1060(\mathrm{C}- \\
\mathrm{S}-\mathrm{C})\end{array}$ & $\begin{array}{l}12.49(\mathrm{~s}, 1 \mathrm{H}, \mathrm{OH}), 8.81(\mathrm{~s}, 3 \mathrm{H}, 3 \mathrm{X} \mathrm{NH}) \\
8.46(\mathrm{~s}, 1 \mathrm{H}, \mathrm{CH}), 7.11-8.12(\mathrm{~m}, 7 \mathrm{H}, \mathrm{CH}- \\
\mathrm{Ar}), 4.02\left(\mathrm{~s}, 3 \mathrm{H}, \mathrm{OCH}_{3}\right), 3.45(\mathrm{~s}, 2 \mathrm{H}, \\
\left.\mathrm{CH}_{2}\right), 2.58\left(\mathrm{~s}, 3 \mathrm{H}, \mathrm{CH}_{3}\right)\end{array}$ \\
\hline 10 & $\begin{array}{l}3350(\mathrm{NH}), 3039(\mathrm{C}-\mathrm{H} \text { aromatic }) \\
1688(\mathrm{C}=\mathrm{N}), 1676(\mathrm{C}=\mathrm{O}), 1537(\mathrm{C}-\mathrm{C} \\
\text { of aromatic ring }), 1065(\mathrm{C}-\mathrm{S}-\mathrm{C})\end{array}$ & $\begin{array}{l}8.85(\mathrm{~s}, 3 \mathrm{H}, 3 \mathrm{X} \mathrm{NH}), 8.44(\mathrm{~s}, 1 \mathrm{H}, \mathrm{CH}), \\
7.13-8.15(\mathrm{~m}, 8 \mathrm{H}, \mathrm{CH}-\mathrm{Ar}), 3.87(\mathrm{~s}, 6 \mathrm{H}, \\
\left.\mathrm{N}\left(\mathrm{CH}_{3}\right)_{2}\right), 3.45\left(\mathrm{~s}, 2 \mathrm{H}, \mathrm{CH}_{2}\right), 2.52(\mathrm{~s}, 3 \mathrm{H}, \\
\left.\mathrm{CH}_{3}\right)\end{array}$ \\
\hline
\end{tabular}

Table 3. Antimicrobial activity of the compounds 2-10

\begin{tabular}{|c|c|c|c|c|c|c|c|}
\hline \multirow{2}{*}{$\begin{array}{c}\text { Compd. } \\
\text { No. }\end{array}$} & \multirow[t]{2}{*}{$\mathrm{R}$} & \multicolumn{3}{|c|}{$\begin{array}{c}\text { Bacterial growth } \\
\text { inhibition(diameter in } \mathrm{mm} \text { ) }\end{array}$} & \multicolumn{3}{|c|}{$\begin{array}{l}\text { Fungi growth inhibition } \\
\text { (diameter in } \mathrm{mm} \text { ) }\end{array}$} \\
\hline & & E. coli & S. aureus & P. vulgaris & A. niger & A. flavus & C. krusai \\
\hline 2 & - & 10 & 8 & - & 7 & - & 9 \\
\hline 3 & - & - & 10 & 8 & - & 8 & - \\
\hline 4 & $\mathrm{H}$ & 12 & - & - & 10 & 12 & 7 \\
\hline 5 & $2-\mathrm{OH}$ & - & 11 & 13 & 11 & 14 & - \\
\hline 6 & $3-\mathrm{OH}$ & 16 & 14 & 15 & 13 & - & 11 \\
\hline 7 & $2-\mathrm{OCH}_{3}$ & 18 & 16 & - & 18 & 25 & 16 \\
\hline 8 & $4-\mathrm{OCH}_{3}$ & 20 & 18 & 16 & 15 & 23 & 14 \\
\hline 9 & $\begin{array}{l}3-\mathrm{OCH}_{3} \\
\& 4-\mathrm{OH}\end{array}$ & 24 & 21 & 22 & 23 & 29 & 19 \\
\hline 10 & $\mathrm{~N}\left(\mathrm{CH}_{3}\right)_{2}$ & 22 & 19 & 20 & 21 & 27 & 18 \\
\hline \multicolumn{2}{|c|}{ Ciprofloxacin } & 22 & 20 & 20 & - & - & - \\
\hline \multicolumn{2}{|c|}{ Fluconazole } & - & - & - & 22 & 30 & 19 \\
\hline
\end{tabular}

\section{Conclusion}

The successful synthesis of series of heterocyclic compounds and evaluation of the antimicrobial activity of thiazole derivatives were reported. From the results of the antimicrobial activity is due to presence of thiazole ring in the structure. Presence of $3-\mathrm{OCH}_{3}$ and 4-OH substituted aniline ring in thiazole derivatives showed better antibacterial as well as antifungal activity. 


\section{Acknowledgement}

We are thankful to SAIF Punjab University, Chandigarh India for spectral and analytical analysis of newly synthesized compounds. We are also thankful to Department of Microbiology LLRM Medical College Meerut UP for their antimicrobial activity.

\section{References}

1. Bondock S, Fadaly W and Metwally M A, Eur J Med Chem., 2010, 45(9), 36923701; DOI:10.1016/j.ejmech.2010.05.018

2. Idhayadhulla A, Kumar R S and Nasser A J A, J Mex Chem Soc., 2011, 55(4), 218-223.

3. Desai N C, Joshi V V, Rajpara K M, Vaghani H V and Satodiya H M, Indian J Chem., 2013, 52B(9), 1191-1201.

4. Gaikwad N D, Patil S V and Bobade V D, J Heterocycl Chem., 2013, 50(3), 519-527; DOI:10.1002/jhet.1513

5. Mishra R, Tomar I, Priyanka, Sharma N K and Jha K K, Der Pharmacia Sinica, 2012, 3(3), 361-366.

6. Hassan A A, Ibrahim Y R, El-Sheref E M, Aziz M A, Brase S and Nieger M, Archiv Der Pharmazie, 2013, 346(7), 562-570; DOI:10.1002/ardp.201300099

7. Bhuiyan M M H and Mustafa Kamal A S M, Chem J., 2012, 2(1), 21-25.

8. Shastri R, World Gournal of Pharmacy and Pharmaceutical Sciences, 2014, 3(7), 1814-1823.

9. Rohini R M and Manjunath M, Der Pharma Chemica, 2012, 4(6), 2438-2441.

10. Arshad M F, Siddiqui N, Elkerdasy A, Al-Rohaini A H and Khan S A, Am J Pharm Toxicol., 2014, 9(2), 132-138; DOI:10.3844/ajptsp.2014.132.138

11. Patel A D and Patel C N, Int J Drug Develop Res., 2012, 4(1), 106-111.

12. Muralikrishna S, Raveendrareddy P, Ravindranath L, Harikrishna and Jagadeeswara Rao P, Der Pharma Chemica, 2013, 5(6), 87-93.

13. Paul S S P, Yardily A, Rajasekharan K N and Abbs Fen Rigi T F, Indian J Chem., 2013, 52B, 560-564.

14. Smith Q E, In pharmacological Screening tests progress in medicinal chemistry, Butterworths London, 1960, 1, 1-33.

15. Pai S T and Platt M W, Letters Applied Microbiology, 1995, 20(1), 14-18;

DOI:10.1111/j.1472-765X.1995.tb00397.x 